\title{
Evaluating and Forecasting Room Demand in Tourist Spot using Holt-Winters Method
}

\author{
C. Premila Rosy \\ PhD Research Scholar, \\ Department of Computer science, \\ Bharathiyar University, \\ Coimbatore -641046
}

\author{
R. Ponnusamy, PhD \\ Prof. of Computer Science \& engineering, \\ Sri Lakshmi Ammaal Engineering college, \\ Thiruvanchery, Chennai - 600126
}

\begin{abstract}
Economic system of any field trend to be dynamic which sometimes leads to fault promotion and worst decision and costly in financial terms. This challenge in financial terms forecasting is highly adopted in many industry. The uncertainty in economic variable is evaluated by instrumental activities in organization. In this research by Holt - Winter method is applied for hotel industry to forecast the room availability. The problem in forecast is based on arrival day room demand may vary which leads to uncertainty. Forecasting is useful widely for hotel management in terms of revenue and provides excellent decision making system for customer and demand. Forecasting method used in this research is quantitative method and does not involves management expertise. Results illustrate forecasting mechanism based on actual data collected from hotel.
\end{abstract}

\section{Keywords}

Forecasting, Hotel rooms, Holt - Winter Method

\section{INTRODUCTION}

Forecasting space demand may be a important a part of modern-day edifice revenue management systems. the target of those systems is to maximize revenue given fixed capability and differing random disposition to pay among market segments. to the current finish, most hotels have enforced some type of inventory controls to make your mind up dynamically that market segments to sell. These improvement routines area unit administrated over many days before the arrival day, thus hotel estimate of the demand for rooms for that exact target day is needed to hold out the improvement. This paper deals with the matter of statement at liberty chamber demand. at liberty space demand is that the range of rooms that may be rented if there are not any capability or evaluation constraints. space allocation and improvement area unit separate problems and aren't self-addressed during this paper.

Optimization of the inventory is extremely vital to the revenue management system. The improvement drawback involves mercantilism the correct form of space to the correct customer at the correct worth, with the target of increasing the revenue. This makes statement a crucial issue, since a far better forecast would end in improved inventory improvement, and consequently, increased revenue. Indeed, statement and improvement are among the first elements of the yield management system [1], and each elements area unit important for the performance of the system. A lot of the work done on edifice revenue management systems deals with the improvement drawback [2, 3, 41.There has but, been very little or no printed work on the statement side. during this paper, we tend to show however a particular statement procedure may be applied to the hotel room demand drawback. Methods used for statement knowledge in business applications include regression techniques, structural time series models and Box-Jenkins models $[5,6,71$. These models but, area unit terribly sophisticated and tough to implement. Smoothing strategies, on the opposite hand, are straightforward and provides equivalent performance with the right alternative of model. Smoothing procedures discount past observations in predicting future knowledge, but the manner during which past knowledge is discounted is adhoc [6].The simple exponential smoothing technique forecasts future knowledge supported past observations [9]. In this method, recent observations area unit given a lot of weights and observations additional within the past area unit given less weight. A a lot of general variation of the exponential smoothing procedure is that the Holt-Winters technique [lo].The latter considers the native linear trend and seasonality in the knowledge. This technique owes its quality to the fact that it's terribly straightforward to implement and is comparable with the other univariate statement procedure in terms of accuracy [11]. Also, the elements of the forecast (viz. mean, trend and seasonality) lend themselves to a simple interpretation. during this paper, we apply the Holt-Winters procedure to forecast at liberty room demand for hotel actual edifice. knowledge collected from an actual edifice is employed within the initialization of the forecast components.

The objective of this paper is to use and appraise the Holt-Winters procedure to the forecast of chamber demand, supported exhausting knowledge solely. during this paper, no human input is accounted for within the forecast mechanism. The current study of an current analysis aiming at developing hotel intelligent system that uses each hard knowledge and human input to get forecast.

\subsection{Forecast Component}

The forecasted worth of demand is comprised of 2 components: the long run and therefore the short term forecasts. The final forecast may be a weighted combination of these 2 elements.

\subsection{Long Term Forecast}

The long run forecast (LTF) estimates the demand for future arrival dates supported historical knowledge. The forecast is also created the maximum amount as a year prior the arrival date, and then it's known as the LTF. We use the Holt-Winters method (described in Section 3) for this forecast.

\subsection{Short Term Forecast}

The short term forecast (STF) is hotel estimate of at liberty net demand for future dates supported the actual advance booking activity. Typically, most of the advance booking requests area unit received throughout the 60 days before the arrival day; therefore the name STF. The procedure for computing the STF is represented in section. 


\section{FORECAST WEIGHTS \& COMBINED FORECAST}

In final stage of forecast the predicted value is obtained by taking a weighted add of the long run and therefore the short term forecasts. The objective is to allow the LTF a better weight than the STF once the LTF can predict higher. The weights are normalized, i.e. their add is unity. During every process day $\mathrm{P}$, mean sq. errors(MSE) of the forecast and therefore the actual worth area unit computed for LTF and STF elements [13]. The weights are computed and updated as follows:

$$
\begin{gathered}
w_{t P}=\frac{\text { Short -TermMSE }}{\text { Short-TermMSE + Long-TermMSE }} \\
w_{t P}=\eta w t_{P t-t}+(1-\eta) w_{t P}
\end{gathered}
$$

The parameter $0<\eta<1$ is mounted indiscriminately, depending on what proportion importance we tend to want to allow to the new weights. For this drawback, $\eta$ is chosen to be adequate to0.9. the ultimate combined forecast is calculated for every arrival day as

$$
\text { FinalForecast }=w_{t P} * L T F+\left(1-w_{t P}\right) * S T F
$$

\section{HOLT-WINTERS TECHNIQUE FOR LONG TERM FORECASTING}

The Holt-Winters technique is hotel extension of the exponentially weighted moving average (EWMA) procedure. The EWMA algorithmic program forecasts future values based on past observations, and places a lot of weight on recent observations. The makeup of the Holt-Winters procedure is that it incorporates linear trend and seasonality into the easy exponential smoothing algorithm [6]. betting on however the seasonal variation is enclosed within the model, there area unit 2 versions of the Holt-Winters forecast procedure: the additive model and therefore the increasing model. Here, we adopt the increasing model given by

$$
y_{t}=\left(m_{t}+b_{t} t\right) c_{t}+\epsilon_{t}
$$

where $m_{t}$ is that the mean part that provides the amount of the statistic at time instant $t, b_{t}$ is that the trend part that indicates the direction during which the series is evolving, $c_{t}$ is that the seasonal part that indicates the periodic variations within the level of the series and $e_{t}$ is the random error part. The update equations are

$$
\begin{aligned}
& \hat{m_{t}}=\frac{\alpha y_{t}}{C_{t-s}}+(1-\alpha)\left(\hat{m_{t-1}}+\hat{b_{t-1}}\right) \\
& \hat{b_{t}}=\beta\left(\hat{m_{t}}-\hat{m_{t-1}}\right)+(1+\beta) \hat{b_{t-1}} \\
& \hat{c_{t}}=\frac{\gamma y_{t}}{\wedge}+(1-\gamma) \hat{\imath} \\
& m_{t}
\end{aligned}
$$

Where $\alpha, \beta$ and $Y$ area unit the smoothing constants for the base, trend and seasonal elements. The forecast for any future time $\mathrm{Y}=1,2, \ldots$ is given by

$$
\hat{y_{t+\gamma}}=\left(\hat{m_{t}}+\hat{b_{t \tau}}\right) \hat{c}_{t+r-s}
$$

As seen from equations four through eight, it's quite simple to implement the Holt-Winters technique on a digital system. we've got used the increasing version to forecast space demand, supported the idea that the seasonal effects area unit proportional in size to the local mean. Initially, the smoothing constants area unit assigned values indiscriminately, and a is optimized before every forecast run to reduce the foremost recent forecast error.

\section{BOOKING PROFILE OF HOTEL USING STF}

The STF uses the advance reservations, cancellation rate, internet turn downs and therefore the net demand booking profile to get estimate of the ultimate demand. The net demand booking profile is obtained from historical data, and it shows the build of reservations for that particular market segment/day of week combination from knowledge of previous years.

$$
S T F=\frac{\text { (net reservations }+ \text { est. net turndowns) }}{\% \text { net demand bkd so far (historical) }}
$$

Where; net reservations $=$ reservations control $\mathrm{x}$ cancellation rate

The net reservations control offers in hotel estimate of the full number of outstanding reservations that area unit expected to show the arrival day. The calculable web turn downs need to be enclosed within the STF algorithmic program since the net reservations don't contain any data regarding reservation requests that were denied because of capability or different constraints. These "turn downs" or denied requests ought to be additional to cyber web reservations since the target is to forecast at liberty demand. Finally, this add is split by the historical fraction of reservations created up-to the process day, therefore extrapolating the demand to the arrival day.

\section{GENERATING RESERVATION AND CANCELLATION REQUESTS}

A reservation request is characterized by 3 quantities: the arrival day, market section or rate class and the length of keep. Likewise, cancellations of some existing reservations also are characterized on similar-lines. to boot, it's vital to understand once the cancellation occurred, i.e. however way before the arrival date was the reservation canceled. As seen in Section4, this affects the short term demand forecast.

In this paper, simulations area unit administrated for one market segment. Extending this work to forecast at liberty demand for many market segments is easy. Forecast algorithmic program having seen the various elements of the forecast, we can currently study the particular procedure used. The forecast algorithm may be simply understood with the assistance of the flow chart shown in Figure 1.The flow chart shows however the 2 forecast elements are calculated and combined to allow the ultimate forecast. Initialization of the long run part involves setting the values of the mean, trend and seasonal elements. The next step within the LTF is to search out the best value of the smoothing parameter.

The value of a is optimized to sight the presence of non-random effects within the knowledge. we tend to begin with initial values of $\mathrm{a}=$ zero. $2, \beta=0.05$ and $\mathrm{y}=$ zero. 1 . Once the values of the smoothing constants area unit mounted, the LTF is computed for all future arrival days. The initialization for the STF includes of determinative the cancellation rates from the historical knowledge. We assume that the 
cancellation rates don't amendment from year to year. The STF is then computed as explained in Section four. Once the long run and short term forecasts area unit generated, the MSE for individual elements area unit calculated and the forecast weights and therefore the final forecast

\section{SIMULATION RESULT AND DISCUSSION}

For data format and simulation functions, fifty eight weeks of data from hotel actual edifice property were used. Data from the primary fifty two weeks area unit used for data format of the forecast parameters, and information from the subsequent6 weeks area unit accustomed generate random reservation and cancellation requests. Figure three shows the particular buildup of reservations, the combined forecast and its parts for a weekday(Test Day 1) within the last week of the simulation amount. The forecast of demand for a selected arrival day is done nightly before the arrival day. For this instance, the forecast starts thirty eight nights before the arrival day. In Figure, the combined forecast and its 2 parts give estimates of the demand up to at some point before the arrival day.

$$
M A P E=\frac{\sum \frac{\left[\text { demand }_{\text {act }}-\text { demand }_{f c s t}\right]}{\text { demand }_{\text {act }}}}{\text { No.ofdays }}
$$

The arrival day corresponds to the zero coordinate on the coordinate axis. the ultimate forecasted values lie on the vertical line drawn at at some point before the arrival day. the purpose at that the particular demand curve meets the coordinate axis provides the worth of the amount of reservations that showed the arrival day.

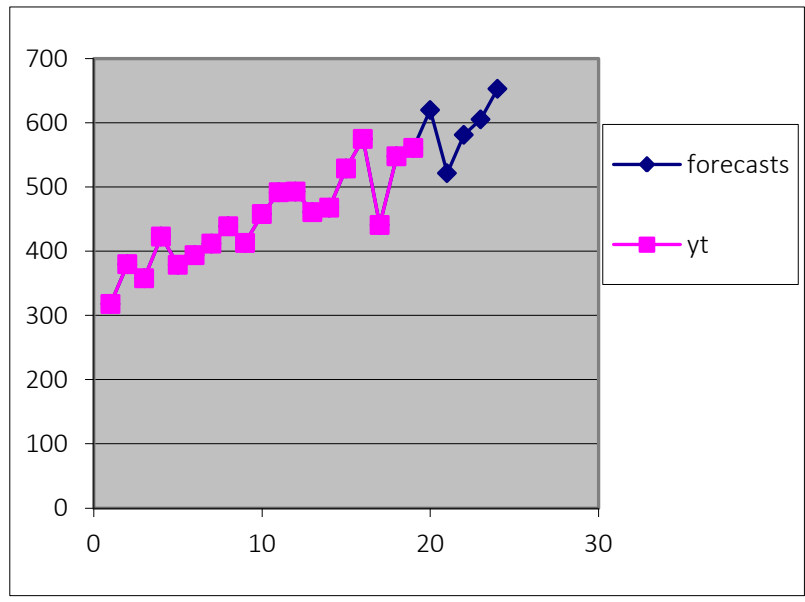

Fig:1 - Comparision of forecasting

As seen from the figure, the combined forecast follows the LTF closely once the arrival day is much from the day the forecast is formed. Initially, the amount of actual reservations and also the historical fraction of demand booked so far is incredibly tiny, leading to a really high worth of the STF. because the arrival day approaches, the speed of reservation requests will increase, and also the STF provides higher estimates. The combined forecast, being a weighted sum of the 2 forecasts, lies in between the 2 estimates. Eventually, the combined forecast provides a satisfactory estimate of the ultimate demand. Figure four shows the same graph for check Day a pair of within the last week of the simulation amount.
The same as the previous case, the forecast for this arrival day starts forty nights before the arrival day. The results obtained within the second action at law area unit similar to those obtained within the initial. we have a tendency to get a decent estimate of the free space demand during this case as well.

During this case, however, there is a large deviation of the forecasted worth from the particular value. the explanations for this behavior of the forecast area unit discussed later within the section. The forecast for at the moment started thirty six nights before the arrival day. From the higher than results, we have a tendency to see that neither the LTF nor the STF will provides a cheap estimate of the demand individually, however a weighted total seems to be effective in some cases. This provides the motivation for the utilization of an extended term - short term forecast methodology. Monte Carlo simulations are accustomed compare the actual and forecasted demand. This was necessary since the reservation and cancellation requests area unit generated randomly. during this paper, the particular and forecasted demand are averaged over one hundred runs for each arrival day. The mean absolute deviation (MAD) is employed as a live of performance of the forecast rule. This is calculated over the last a pair of weeks of the simulation amount. We think about check days within the last a pair of weeks of the simulation amount since we are able to observe however the forecast develops over a amount of at least thirty days.

\section{CONCLUSION}

This paper mentioned the Holt-Winters prognostication procedure and its application to prognostication free hotel room demand. The parameters of the Holt-Winters model area unit initialized exploitation historical information obtained from hotel actual edifice. The Holt-Winters forecast approach was accustomed cipher the LTF of space demand. The STF was computed supported actual booking activity. the ultimate forecast could be a weighted total of the long term and also the short term forecasts, and also the forecast weights area unit determined by the mean sq. error between the forecasted and also the actual values. As mentioned within the starting of the paper, we have a tendency to feel that a decent forecast can lead to better inventory improvement and management. Thus, the objective is to be ready to get a decent forecast consistently for all future days, instead of having hotel exact prediction of the demand on some days. This logically takes America to the utilization of symbolic logic within the forecast algorithm. These ideas area unit being investigated and that they certainly gift a brand new approach of confronting the matter.

\section{REFERENCES}

[1] Cross, R. G., Higbie, J. A., \& Cross, Z. N. (2011). Milestones in the application of analytical pricing and revenue management. Journal of Revenue and Pricing Management, 10(1), 8-18.

[2] Bitran, G. R., \& Gilbert, S. M. (1996). Managing hotel reservations with uncertain arrivals. Operations Research, 44(1), 35-49.

[3] Bitran, G. R., \& Mondschein, S. V. (1995). An application of yield management to the hotel industry considering multiple day stays. Operations research, 43(3), 427-443.

[4] Ladany, S. P. (1976). Dynamic operating rules for motel reservations. Decision Sciences, 7(4), 829-840. 
[5] Montgomery, D. C., Johnson, L. A., \& Gardiner, J. S. (1990). Forecasting and time series analysis. McGraw-Hill Companies.

[6] Harvey, A. C. (1993). Time Series Models MIT Press. Cambridge, Massachusetts, USA.

[7] Akaike, H. (1976). Canonical correlation analysis of time series and the use of an information criterion. Mathematics in Science and Engineering, 126, 27-96.

[8] Disney, S. M., \& Lambrecht, M. R. (2008). On replenishment rules, forecasting, and the bullwhip effect in supply chains. Foundations and Trends 庐 in
Technology, Information and Operations Management,2(1), 1-80.

[9] Chatfield, C. (2016). The analysis of time series: an introduction. CRC press.

[10] Chatfield, C. (1978). The holt-winters forecasting procedure. Applied Statistics, 264-279.

[11] Harvey, A. C. (1990). Forecasting, structural time series models and the Kalman filter. Cambridge university press.

[12] Rajopadhye, M., Ghalia, M. B., Wang, P. P., Baker, T., \& Eister, C. V. (2001). Forecasting uncertain hotel room demand. Information Sciences, 132(1), 1-11. 